\title{
Prostate Saturation Biopsy
}

National Cancer Institute

\section{Source}

National Cancer Institute. Prostate Saturation Biopsy. NCI Thesaurus. Code C113288.

Transperineal prostate biopsy that removes more than 20 cores. This is typically reserved for high-risk patients. 